\title{
SEQUENCE OF REGULAR FINITELY ADDITIVE SET FUNCTIONS(1)
}

\author{
BY
}

\author{
J. D. STEIN, JR.
}

\begin{abstract}
The purpose of this paper is to deduce versions of Phillips' lemma and the Vitali-Hahn-Saks theorem, with weaker conditions placed on the set functions and convergence conditions than is usually required.
\end{abstract}

Let $X$ be a topological space, $\mathscr{B}$ the $\sigma$-field of the Borel sets of $X$. A bounded finitely additive set function $\mu$ defined on $(X, \mathscr{B})$ is said to be regular if, for each $\varepsilon>0$ and $E \in \mathscr{B}$, there is a compact set $K \subseteq E$ such that $|\mu(E \sim K)|<\varepsilon$. Throughout this paper, all set functions will be assumed to be bounded, finitely additive, and regular. If $\mu$ is a set function, $|\mu|$ will denote its total variation.

We shall be interested in propositions of the following type: let $\Sigma$ be a subclass of $\mathscr{B}$, and let $\left\{\mu_{n} \mid n=1,2, \ldots\right\}$ be a sequence of set functions defined on $(X, \mathscr{B})$ such that, for each $E \in \Sigma$, the sequence of complexes $\left\{\mu_{n}(E) \mid n=1,2, \ldots\right\}$ satisfies a convergence condition of some sort. We wish to deduce, if possible, stronger convergence properties of $\left\{\mu_{n} \mid n=1,2, \ldots\right\}$.

Two well-known theorems of this type are Phillips' lemma [1, Lemma 3.3] and the Vitali-Hahn-Saks theorem. For both of these theorems, $\Sigma=\mathscr{B}$. In Phillips' lemma, the topology of $X$ is quite restrictive. We shall show that, if the set functions are assumed to be regular, then results similar to Phillips' lemma and the Vitali-Hahn-Saks theorem can be obtained when either the class of all open sets or the class of all $\sigma$-compact sets (countable unions of compact sets) is used in the hypotheses of the propositions.

1. Three preliminary lemmas. The following three lemmas will be quite useful throughout the remainder of this paper.

Lemma 1.1. If $\mu$ is a bounded regular finitely-additive set function, then so is $|\mu|$.

Proof. We need merely show $|\mu|$ is regular. Let $E$ be a Borel set, $\varepsilon>0$. Let $E_{1}, \ldots, E_{n}$ be a partition of $E$ such that $\sum_{k=1}^{n}\left|\mu\left(E_{k}\right)\right|>|\mu|(E)-\varepsilon / 2$. By regularity of $\mu$, choose compact sets $K_{1}, \ldots, K_{n}$ such that $K_{j} \subseteq E_{j}$ for $1 \leq j \leq n$ and $\left|\mu\left(E_{j} \sim K_{j}\right)\right|<\varepsilon / 2 n$ for $1 \leq j \leq n$. Let $K=\bigcup_{j=1}^{n} K_{j}$; then $K$ is compact. Also,

$$
\begin{aligned}
|\mu|(E) & <\frac{\varepsilon}{2}+\sum_{j=1}^{n}\left|\mu\left(E_{j}\right)\right| \leq \frac{\varepsilon}{2}+\sum_{j=1}^{n}\left|\mu\left(E_{j} \sim K_{j}\right)\right|+\sum_{j=1}^{n}\left|\mu\left(K_{j}\right)\right| \\
& <\varepsilon+|\mu|(K),
\end{aligned}
$$

which completes the proof. Q.E.D.

Received by the editors September 30, 1972.

AMS (MOS) subject classifications (1970). Primary 46G99.

Key words and phrases. Functional analysis, measure theory.

(1) This work was supported by NSF GP-29011 and NSF GP-33693X. 
Lemma 1.2. Let $\mu$ be a bounded regular finitely additive set function.

(a) If $E$ is a Borel set, then there is a compact set $K \subseteq E$ such that $|\mu(K)|$ $>|\mu|(E) / 7$, if $|\mu|(E) \neq 0$.

(b) If $U$ is an open set such that $|\mu|(U)>0$, then there is an open set $V \subseteq U$ with $|\mu(V)|>|\mu|(U) / 7$.

Proof. See [2, Lemma 1] for details. Q.E.D.

Lemma 1.3. Let $\mu$ be a regular bounded finitely additive set function, and let $\left\{F_{n} \mid n=1,2, \ldots\right\}$ be a sequence of Borel sets, $\left\{E_{n} \mid n=1,2, \ldots\right\}$ a sequence of disjoint open sets such that $F_{n} \subseteq E_{n}$ for $n=1,2, \ldots$ Then $\left|\mu\left(\cup_{n=1}^{\infty} F_{n}\right)\right|$ $\leq \sum_{n=1}^{\infty}|\mu|\left(F_{n}\right)$.

Proof. Let $\varepsilon>0$; by regularity of $\mu$ we can find a compact set $K \subseteq \cup_{n=1}^{\infty} F_{n}$ such that $\left|\mu\left(\cup_{n=1}^{\infty} F_{n} \sim K\right)\right|<\varepsilon$. Since $K$ is compact, $K \subseteq \cup_{n=1}^{N} E_{n}$ for some integer $N$, and so $K \subseteq \cup_{n=1}^{N} F_{n}$. Since $F_{1}, \ldots, F_{N}$ are disjoint we have

$$
\begin{aligned}
\left|\mu\left(\bigcup_{n=1}^{\infty} F_{n}\right)\right| & \leq\left|\mu\left(\bigcup_{n=1}^{\infty} F_{n} \sim K\right)\right|+|\mu(K)|<\varepsilon+|\mu|(K) \\
& \leq \varepsilon+\sum_{n=1}^{N}|\mu|\left(F_{n}\right) \leq \varepsilon+\sum_{n=1}^{\infty}|\mu|\left(F_{n}\right) .
\end{aligned}
$$

Since $\varepsilon>0$ was arbitrary, the lemma follows. Q.E.D.

2. Existence of maximal open sets. Our goal in this section is to prove two similar propositions for versions of Phillips' lemma and the Vitali-Hahn-Saks theorem. We first prove a version of Phillips' lemma.

Proposition 2.1. (a) Let $X$ be a $T_{1}$ and $T_{3}$ topological space, $\left\{\mu_{n} \mid n=1,2, \ldots\right\}$ a sequence of bounded regular finitely additive set functions defined on the Borel sets of $X$. Suppose that $\mu_{n}(E) \rightarrow 0$ for each open set $E$. Then there exists a maximal open set $U$ such that $\left|\mu_{n}\right|(U) \rightarrow 0$.

(b) If we assume that $X$ is $T_{2}$ and $\mu_{n}(E) \rightarrow 0$ for each $\sigma$-compact set $E$, a similar conclusion can be obtained.

Proof. We shall merely prove (a), as the proof of (b) is similar. Let $\mathcal{E}$ $=\left\{E \subseteq X \mid E\right.$ is open and $\left.\left|\mu_{n}\right|(E) \rightarrow 0\right\}$ and let $U=\cup\{E \mid E \in \varepsilon\}$. We shall show $U \in \mathcal{E}$ to complete the proof.

If $U=\varnothing$, there is clearly nothing to prove. If $\left|\mu_{n}\right|(U) \nrightarrow 0$, then there is an $\varepsilon>0$ and a subsequence $\left\{\mu_{n_{k}} \mid k=1,2, \ldots\right\}$ such that $\left|\mu_{n_{k}}\right|(U)>\varepsilon$. Since $\left|\mu_{n_{1}}\right|(U)>\varepsilon / 2$, by Lemma 1.2 we can find an open set $V_{1} \subseteq U$ such that $\left|\mu_{n_{1}}\left(V_{1}\right)\right|>\varepsilon / 14$. We can find, for any $\Re>0$, a compact set $K_{1} \subseteq V_{1}$ such that $\left|\mu_{n_{1}}\right|\left(V_{1} \sim K_{1}\right)<\vartheta$. Since $K_{1} \subseteq U$ and $K_{1}$ is compact, $K_{1}$ can be covered by a finite subcover selected from $\delta$; by intersecting the members of this subcover with $V_{1}$ we can obtain an open set $E_{1}$ such that $K_{1} \subseteq E_{1} \subseteq V_{1}$ and $E_{1} \in \mathcal{E}$ (since $\mathcal{E}$ is clearly closed under finite unions). By the regularity of $X$, we can find an open 
set $O_{1}$ with $K_{1} \subseteq O_{1} \subseteq \bar{O}_{1} \subseteq E_{1}$. By choosing $\Re$ sufficiently small, we can insure that $\left|\mu_{n_{1}}\left(O_{1}\right)\right|>\varepsilon / 14$. Also, since $\left|\mu_{n}\right|\left(\bar{O}_{1}\right) \rightarrow 0$, we can find an integer $p_{1}$ such that $k \geq p_{1} \Rightarrow\left|\mu_{n_{k}}\right|\left(U \sim \bar{O}_{1}\right)>\varepsilon$.

Let $r_{1}=n_{1}$. Suppose that $O_{1}, \ldots, O_{n}$, a collection of open sets with disjoint closures, and $r_{1}<\cdots<r_{n}$ have been chosen, as well as integers $p_{1}, \ldots, p_{n}$ such that

$$
\begin{array}{ll}
\multicolumn{1}{c}{k \geq p_{n} \Rightarrow\left|\mu_{n_{k}}\right|\left(U \sim \bigcup_{j=1}^{n} \bar{O}_{j}\right)>\varepsilon,} \\
\left|\mu_{r_{j}}\left(O_{j}\right)\right|>\varepsilon / 14 & \text { for } 1 \leq j \leq n, \\
\mid \mu_{r_{j}}\left(O_{k}\right)<\varepsilon / 56 \cdot 2^{k} & \text { for } 1 \leq j<k \leq n, \\
\left|\mu_{r_{j}}\left(O_{k}\right)\right|<\varepsilon / 28(j-1) & \text { for } 1 \leq k<j \leq n .
\end{array}
$$

We wish to show that we can continue this induction, for if we can, we will be able to obtain a contradiction as follows: let $O=\cup_{j=1}^{\infty} O_{j}$. We then have, using (2), (3), and (4) above, that

$$
\begin{aligned}
\left|\mu_{r_{n}}(O)\right| & =\left|\mu_{r_{n}}\left(\bigcup_{j=1}^{\infty} O_{j}\right)\right|=\left|\mu_{r_{n}}\left(O_{n}\right)+\mu_{r_{n}}\left(\bigcup_{j \neq n} O_{j}\right)\right| \\
& \geq\left|\mu_{r_{n}}\left(O_{n}\right)\right|-\left|\mu_{r_{n}}\left(\bigcup_{j \neq n} O_{j}\right)\right| \\
& \geq\left|\mu_{r_{n}}\left(O_{n}\right)\right|-\sum_{j=1}^{n-1}\left|\mu_{r_{n}}\left(O_{j}\right)\right|-\left|\mu_{r_{n}}\left(\bigcup_{j=n+1}^{\infty} O_{j}\right)\right| \\
& \geq\left|\mu_{r_{n}}\left(O_{n}\right)\right|-\sum_{j=1}^{n-1}\left|\mu_{r_{n}}\left(O_{j}\right)\right|-\sum_{j=n+1}^{\infty}\left|\mu_{r_{n}}\right|\left(O_{j}\right) \quad \text { (by Lemma 1.3) } \\
& \geq \frac{\varepsilon}{14}-\sum_{j=1}^{n-1} \frac{\varepsilon}{28(n-1)}-\sum_{j=n+1}^{\infty} \frac{\varepsilon}{56 \cdot 2^{j}} \geq \frac{\varepsilon}{14}-\frac{\varepsilon}{28}-\frac{\varepsilon}{56}=\frac{\varepsilon}{56} .
\end{aligned}
$$

This contradicts the fact that $\mu_{n}(O) \rightarrow 0$, since $O$ is open.

Consequently, suppose $O_{1}, \ldots, O_{n}, p_{1}, \ldots, p_{n}$, and $r_{1}, \ldots, r_{n}$ have been selected to satisfy properties (1)-(4). Since $\lim _{m \rightarrow \infty} \mu_{m}\left(O_{k}\right)=0$ for $1 \leq k \leq n$, choose an integer $q$ so large that $m \geq q \Rightarrow\left|\mu_{m}\left(O_{k}\right)\right|<\varepsilon / 28 n$ for $1 \leq k \leq n$. Let $t_{1}=p_{n}+1$; using Lemma 1.2 again we can find an open $W_{1}$ such that $\left|\mu_{n_{1}}\left(W_{1}\right)\right|>\varepsilon / 14$. Employment of the techniques used in the first part of this proof will enable us to find an open set $Y_{1}$ such that

(a) $\overline{Y_{1}} \cap\left(\cup_{j=1}^{n} \bar{O}_{j}\right)=\varnothing$,

(b) $\overline{Y_{1}} \subset U$,

(c) $\left|\mu_{n_{1}}\left(Y_{1}\right)\right|>\varepsilon / 14$,

(d) $\exists$ an integer $N_{1}$ such that

$$
k \geq N_{1} \Rightarrow\left|\mu_{n_{k}}\right|\left(U \sim\left(\overline{Y_{1}} \cup\left(\bigcup_{j=1}^{n} \bar{O}_{j}\right)\right)\right)>\varepsilon .
$$


Property (d) enables us to continue inductively, choosing integers $t_{1}<\cdots$ $<t_{p}<\cdots$ and open sets $Y_{1}, \ldots, Y_{p}, \ldots$ such that

(a') $\bar{Y}_{p} \cap\left(\cup_{j=1}^{p-1} \bar{Y}_{j} \cup \cup_{k=1}^{n} \bar{O}_{k}\right)=\varnothing$,

(b') $\overline{Y_{p}} \subset U$,

(c') $\left|\mu_{n_{s}}\left(Y_{p}\right)\right|>\varepsilon / 14$,

(d') $\exists$ an integer $N_{p}$ such that

$$
k \geq N_{p} \Rightarrow\left|\mu_{n_{k}}\right|\left(U \sim\left(\bigcup_{j=1}^{p} \bar{Y}_{j} \cup \bigcup_{k=1}^{n} \bar{O}_{k}\right)\right)>\varepsilon .
$$

Choose an integer $p>1+\left(56 \cdot 2^{n+1} / \varepsilon\right) \sum_{j=1}^{n}\left|\mu_{r}\right|(X)$; then some one of the sets $Y_{1}, \ldots, Y_{p}$, call it $Y_{m}$, must satisfy $\left|\mu_{r_{j}}\right|\left(Y_{m}\right)<\varepsilon / 56 \cdot 2^{n+1}$ for $1 \leq j \leq n$. This is clear, since for each $j$ between 1 and $n$, at most $\left(56 \cdot 2^{n+1} / \varepsilon\right)\left|\mu_{r j}\right|(\dot{X})$ of the sets $Y_{1}, \ldots, Y_{p}$ can have $\mu_{r_{j}}$-measure greater than or equal to $\varepsilon / 56 \cdot 2^{n+1}$, as the sets $Y_{1}, \ldots, Y_{p}$ are disjoint. So let $Y_{m}$ be designated as the set $O_{n+1}$, let $\mu_{r_{n+1}}$ be the set function associated with $O_{n+1}=Y_{m}$ by property $\left(\mathrm{c}^{\prime}\right)$, and let the integer $p_{n+1}$ be chosen to be the integer $N_{m}$ associated with $Y_{m}$ by property ( $\left.\mathrm{d}^{\prime}\right)$. This completes the proof. Q.E.D.

We now prove a similar version of the Vitali-Hahn-Saks theorem. The technique employed in Proposition 2.1 is a variation of the "gliding hump" argument employed in [2]; since similar techniques will be used in the next proposition we shall merely outline its proof rather than go into complete detail. We say $\mu \ll \nu$ if, given $\varepsilon>0, \exists \delta>0$ such that $|\nu|(E)<\delta \Rightarrow|\mu(E)|<\varepsilon$.

Proposition 2.2. (a) Let $X$ be $a T_{1}$ and $T_{3}$ topological space, $\left\{\mu_{n} \mid n=1,2, \ldots\right\} a$ sequence of bounded regular finitely additive set functions defined on the Borel sets of $X$. Suppose that $\mu$ is also a bounded regular finitely additive set function such that $\mu_{n} \ll \mu$ for $n=1,2, \ldots$, and that for each open set $E, \lambda(E)=\lim _{n \rightarrow \infty} \mu_{n}(E)$ exists. Then there exists a maximal open set $U$ such that, given $\varepsilon>0$, there is a $\delta>0$ and such that $E \in \mathscr{B}, E \subseteq U,|\mu|(E)<\delta \Rightarrow\left|\mu_{n}(E)\right|<\varepsilon$ for $n=1,2$,

(b) A similar conclusion can be obtained if it is assumed that $X$ is $T_{2}$ and $\lambda(E)=\lim _{n \rightarrow \infty} \mu_{n}(E)$ exists for each $\sigma$-compact Borel set $E$.

Proof. As mentioned before, we shall sketch the proof of (b). We call a set admissible if, given $\varepsilon>0$, there is a $\delta>0$ such that if $E$ is a Borel subset of the admissible set with $|\mu|(E)<\delta$, then $\left|\mu_{n}(E)\right|<\varepsilon$ for $n=1,2, \ldots$. We observe that $F$ is admissible iff, given $\varepsilon>0$, there is a $\delta>0$ such that, if $K \subseteq F$ is compact and $|\mu|(K)<\delta$, then $\left|\mu_{n}(K)\right|<\varepsilon$. Suppose that $F$ has the above property, let $\varepsilon>0$, and choose $\delta$ to correspond to $\varepsilon / 2$. Let $E$ be a Borel set, $E \subseteq F$, and $|\mu|(E)<\delta$. Fix an integer $n$, by regularity of $\mu_{n}$ choose a compact $K \subseteq E$ such that $\left|\mu_{n}(E \sim K)\right|<\varepsilon / 2$. Then $|\mu|(K)<\delta \Rightarrow\left|\mu_{n}(K)\right|<\varepsilon / 2$, and 
so $\left|\mu_{n}(E)\right| \leq\left|\mu_{n}(E \sim K)\right|+\left|\mu_{n}(K)\right|<\varepsilon$. So $F$ is admissible, and the assertion follows.

We say that $F$ is inadmissible for $\varepsilon>0$ if for each $\delta>0$ we can find a Borel set $E \subseteq F$ with $|\mu|(E)<\delta$ and $\left|\mu_{n}(E)\right| \geq \varepsilon$ for an infinite number of subscripts $n$. Our next assertion is that if $U$ is inadmissible for $\varepsilon>0$ and $F \subseteq U$ is admissible, then $U \sim F$ is inadmissible for $\varepsilon-\pi$, where $0<\pi<\varepsilon$. Choose $\delta>0$ such that $K \in \mathscr{B}, K \subseteq F,|\mu|(K)<\delta \Rightarrow\left|\mu_{n}(K)\right|<\Re / 2$. If $K \subseteq U$, we can write $K=(K \cap F) \cup(K \sim F)$. Let $\left\{K_{n} \mid n=1,2, \ldots\right\}$ be Borel sets such that $K_{n} \subseteq U,|\mu|\left(K_{n}\right)<1 / n$, and $\left\{\mu_{n_{k}}^{(n)} \mid k=1,2, \ldots\right\}$ be subsequences such that $\left|\mu_{n_{k}}^{(n)}\left(K_{n}\right)\right|>\varepsilon$. For large $n,|\mu|\left(K_{n} \cap F\right)<\delta$, and so $\left|\mu_{p}\left(K_{n} \cap F\right)\right|<\pi / 2$, for all $p$ and large $n$, consequently $\left|\mu_{n_{k}}^{(n)}\left(K_{n} \sim F\right)\right|>\varepsilon-\Re$ for all $k$ and large $n$. Since $K_{n} \sim F \subseteq U \sim F$, we see that $U \sim F$ is inadmissible for $\varepsilon-\pi$.

Let $U$ denote the union of all admissible open sets. Our goal is to show that $U$ is admissible; this will complete the proof. Suppose that $U$ is inadmissible for some $\varepsilon>0$. Note that any finite union of admissible sets is admissible; and if $K \subseteq U$ is compact, then $K$ is admissible, as by compactness it is contained in a finite union of admissible sets.

In order to obtain a contradiction, we shall choose a disjoint sequence of compact sets $\left\{K_{n} \mid n=1,2, \ldots\right\}$ and a sequence $\left\{\lambda_{n}=\mu_{j(n)} \mid n=1,2, \ldots\right\}$ where $j(n)<j(n+1)$, such that $\left\{\lambda_{n}\left(\cup_{j=1}^{\infty} K_{j}\right) \mid n=1,2, \ldots\right\}$ is not convergent. Our basic tool, derived from Lemma 1.3 and the triangle inequality, is the following inequality:

$$
\begin{aligned}
\left|\left(\lambda_{n+1}-\lambda_{n}\right)\left(\bigcup_{j=1}^{\infty} K_{j}\right)\right| \geq & \left|\lambda_{n+1}\left(K_{n+1}\right)\right|-\left|\lambda_{n}\left(K_{n+1}\right)\right| \\
& -\sum_{j=1}^{n}\left|\left(\lambda_{n+1}-\lambda_{n}\right)\left(K_{j}\right)\right|-\sum_{j=n+2}^{\infty}\left(\left|\lambda_{n+1}\right|+\left|\lambda_{n}\right|\right)\left(K_{j}\right) .
\end{aligned}
$$

Since $U$ is inadmissible for $\varepsilon>0$, we can find a compact $K_{1} \subseteq U$ such that $\left|\mu_{p}\left(K_{1}\right)\right|>\varepsilon / 3$ for an infinite number of subscripts $p$. Let $\left\{\alpha_{n} \mid n=1,2, \ldots\right\}$ be a sequence of constants to be determined later. Since $\lim _{n \rightarrow \infty} \mu_{n}\left(K_{1}\right)=\lambda\left(K_{1}\right)$, let $N(1)$ be such that $n \geq N(1) \Rightarrow\left|\mu_{n}\left(K_{1}\right)-\lambda\left(K_{1}\right)\right|<\alpha_{1} \varepsilon$. Let $\lambda_{1}=\mu_{p}$, where $p$ is the smallest integer greater than or equal to $N(1)$ such that $\left|\mu_{p}\left(K_{1}\right)\right|>\varepsilon / 3$. Let $\left\{\beta_{n} \mid n=1,2, \ldots\right\}$ be another sequence of constants to be determined later.

Suppose now that $\lambda_{1}=\mu_{p_{1}}, \ldots, \lambda_{n}=\mu_{p_{n}}$, disjoint compact sets $K_{1}, \ldots, K_{n}$, and integers $N(1), \ldots, N(n)$ have been chosen such that $k \geq N(j) \Rightarrow \mid \mu_{k}\left(K_{j}\right)$ $-\lambda\left(K_{j}\right) \mid<\alpha_{j} \varepsilon$ for $1 \leq j \leq n$, and that $p_{j} \geq N(j)$ for $1 \leq j \leq n$. We wish to continue the induction in such a way as to use (*) to obtain a contradiction.

Note first that, for any $\pi$ with $0<\Re<\varepsilon, U \sim\left(\cup_{j=1}^{n} K_{j}\right)$ is inadmissible for $\varepsilon-\Re_{\text {. Since }} \lambda_{n} \ll \mu$, for any $\rho>0$ we can find a $\delta_{n}(\rho)>0$ such that, if $E$ is a Borel set with $|\mu|(E)<\delta_{n}(\rho)$, then $\left|\lambda_{n}(E)\right|<\rho$. We now fix $\rho$ (which will, like $\left\{\alpha_{n} \mid n=1,2, \ldots\right\}$ and $\left\{\beta_{n} \mid n=1,2, \ldots\right\}$, be chosen later). We can find a 
compact subset $K_{n+1}^{(1)} \subseteq U \sim\left(\cup_{j=1}^{n} K_{j}\right)$ such that $|\mu|\left(K_{n+1}^{(1)}\right)<\delta_{n}(\rho)$ and $\left|\mu_{r}\left(K_{n+1}^{(1)}\right)\right|$ $>\varepsilon / 3$ for an infinite number of subscripts $r$. Let $I(1)$ be such that $j \geq I(1)$ $\Rightarrow\left|\mu_{j}\left(K_{n+1}^{(1)}\right)-\lambda\left(K_{n+1}^{(1)}\right)\right|<\alpha_{n+1} \varepsilon$. Let $r_{i}$ be the smallest integer greater than or equal to $\max (N(1), \ldots, N(n), I(1))$ such that $\left|\mu_{n}\left(K_{n+1}^{(1)}\right)\right|>\varepsilon / 3$.

Since $U \sim\left(K_{n+1}^{(1)} \cup \cup_{j=1}^{n} K_{j}\right)$ is inadmissible for any $\varepsilon-\Re$ with $0<\Re<\varepsilon$, we can continue this procedure. We thus obtain sequences of disjoint compact sets $\left\{K_{n+1}^{(s)} \mid s=1,2, \ldots\right\}$ and integer sequences $\left\{r_{s} \mid s=1,2, \ldots\right\}$ and $\{I(s) \mid s$ $=1,2, \ldots\}$ such that

(a) $r_{s} \geq \max (N(1), \ldots, N(n), I(s))$

(b) $j \geq I(s) \Rightarrow\left|\mu_{j}\left(K_{n+1}^{(s)}\right)\right|<\alpha_{n+1} \varepsilon$,

(c) $|\mu|\left(K_{n+1}^{(s)}\right)<\delta_{n}(\rho)$,

(d) $\left|\mu_{r_{3}}\left(K_{n+1}^{(s)}\right)\right|>\varepsilon / 3$,

(e) $K_{n+1}^{(s)} \subseteq U \sim\left(\cup_{j=1}^{n} K_{j} \cup \bigcup_{i=1}^{s=1} K_{n+1}^{(l)}\right)$.

By an exhaustion argument similar to that employed in the proof of Proposition 2.1, there is an integer $s_{0}$ such that $\left|\lambda_{j}\right|\left(K_{n+1}^{\left(s_{0}\right)}\right)<\beta_{n+1} \varepsilon$ for $1 \leq j \leq n$. By (c), $|\mu|\left(K_{n+1}^{\left(s_{0}\right)}\right)<\delta_{n}(\rho) \Rightarrow\left|\lambda_{n}\left(K_{n+1}^{\left(s_{0}\right)}\right)\right|<\rho$. Let $K_{n+1}=K_{n+1}^{\left(s_{0}\right)}, \quad \lambda_{n+1}=\mu_{n_{n},}$, and let $N(n+1)=I\left(s_{0}\right)$. If $1 \leq j \leq n$ we have

$$
\left|\lambda_{n+1}\left(K_{j}\right)-\lambda_{n}\left(K_{j}\right)\right| \leq\left|\lambda_{n+1}\left(K_{j}\right)-\lambda\left(K_{j}\right)\right|+\left|\lambda\left(K_{j}\right)-\lambda_{n}\left(K_{j}\right)\right| \leq 2 \alpha_{j} \varepsilon
$$

by the induction hypothesis and properties (a) and (b) above. Accumulating all this information and applying it to (*) yields

$$
\left|\left(\lambda_{n+1}-\lambda_{n}\right)\left(\bigcup_{j=1}^{\infty} K_{j}\right)\right| \geq \frac{\varepsilon}{3}-\rho-\sum_{j=1}^{n} 2 \alpha_{j} \varepsilon-\sum_{j=n+2}^{\infty} 2 \beta_{j} \varepsilon .
$$

Letting $\rho=\varepsilon / 12$ and $\alpha_{j}=\beta_{j}=1 / 24 \cdot 2^{j}$ for $j=1,2, \ldots$, we obtain

$$
\left|\left(\lambda_{n+1}-\lambda_{n}\right)\left(\bigcup_{j=1}^{\infty} K_{j}\right)\right| \geq \frac{\varepsilon}{3}-\frac{\varepsilon}{12}-\sum_{j=1}^{\infty} \frac{\varepsilon}{12 \cdot 2^{j}} \geq \frac{\varepsilon}{6} .
$$

So $\left\{\mu_{n}\left(\cup_{j=1}^{\infty} K_{j}\right) \mid j=1,2, \ldots\right\}$ cannot be Cauchy, and this contradiction establishes the theorem. Q.E.D.

In examining the proofs of Proposition 2.1 and 2.2, we observe that the critical element of both proofs is to show that compact sets are, in a sense determined by the desired conclusion, good. This gives rise to the following two propositions, which we state without proof.

Proposition 2.3. Assume that the hypotheses of either Proposition 2.1(a) or Proposition 2.1(b) hold and that, in addition, $\lim _{n \rightarrow \infty}\left|\mu_{n}\right|(K)=0$ for each compact set $K$. Then $\lim _{n \rightarrow \infty}\left|\mu_{n}\right|(X)=0$.

Proposition 2.4. Assume that the hypotheses of either Proposition 2.2(a) or Proposition 2.2(b) hold and that, in addition, compact sets are admissible. Then $X$ is admissible.

Unfortunately, under general conditions, we cannot show a priori that the 
maximal open sets obtained in Propositions 2.1 and 2.2 are nonempty. However, we have the following proposition.

Proposition 2.5. (a) Suppose the hypotheses of Proposition 2.1(a) hold, and let $\left\{U_{\alpha} \mid \alpha \in \Lambda\right\}$ be an uncountable collection of disjoint open sets. Then $\lim _{n \rightarrow \infty}\left|\mu_{n}\right|\left(U_{\alpha}\right)=0$ for all but a countable subset of $\Lambda$.

for all but a countable subset of $\Lambda$.

(b) Suppose the hypotheses of Proposition 2.1(b) hold, and let $\left\{E_{\alpha} \mid \alpha \in \Lambda\right\}$ be an uncountable collection of disjoint Borel sets. Suppose also that each $\mu_{n}$ is countably additive. Then $\lim _{n \rightarrow \infty}\left|\mu_{n}\right|\left(E_{\alpha}\right)=0$ for all but a countable subset of $\Lambda$.

(c) The analogues of (a) and (b), when applied to Proposition 2.2, also hold.

Proof. (a) Let $\Lambda_{n}=\left\{\alpha \in \Lambda\left|\lim \sup _{k \rightarrow \infty}\right| \mu_{k} \mid\left(E_{\alpha}\right) \geq 1 / n\right\}$. If there are an uncountable collection of subscripts $\alpha$ such that $\lim _{\sup } \operatorname{sum}_{k \rightarrow \infty}\left|\mu_{k}\right|\left(E_{\alpha}\right)>0$, then some $\Lambda_{n}$ must be uncountable. We can therefore find an $\varepsilon>0$ and a disjoint sequence $\left\{E_{n} \mid n=1,2, \ldots\right\}$ of open sets such that, for each $n$,

$$
\lim \sup _{k \rightarrow \infty}\left|\mu_{k}\right|\left(E_{n}\right)>\varepsilon \text {. }
$$

The techniques of Proposition 2.1(a) can now be applied.

(b) This is proved similarly. The hypothesis that each $\mu_{n}$ is countably additive is needed to ensure that Lemma 1.3 can be used without separating the Borel sets by open sets.

(c) is proved similarly. Q.E.D.

Under the hypothesis of Proposition 2.5(a), if $\left\{E_{n} \mid n=1,2, \ldots\right\}$ are disjoint open sets, we can show that, if $\alpha_{k}=\lim \sup _{n \rightarrow \infty}\left|\mu_{n}\right|\left(E_{k}\right)$, that $\lim _{k \rightarrow \infty} \alpha_{k}=0$. The proof is similar.

3. Additional results. All the results of this section will be variations of the Phillips' lemma theme. Although parallel results can be deduced for the VitaliHahn-Saks situation, they are of more interest in the Phillips' lemma setting.

Proposition 3.1. Let $X$ be $a T_{1}$ and $T_{3}$ topological space, and let $\left\{\mu_{n} \mid n=1,2, \ldots\right\}$ be a sequence of regular finitely additive bounded set functions defined on 9 such that $\mu_{n}(E) \rightarrow 0$ for every open set $E$. Let $x \in X$, and let $\varepsilon>0$. Then there is a neighborhood $U(x, \varepsilon)$ of $x$ and an integer $N(x, \varepsilon)$ such that $n \geq N(x, \varepsilon) \Rightarrow\left|\mu_{n}\right|$ $(U(x, \varepsilon))<\varepsilon$.

Proof. As in the proof of Proposition 2.1(a), we need only demonstrate how the induction proceeds. If the conclusion is false, there is a subsequence $\left\{\mu_{n_{k}} \mid\right.$ $k=1,2, \ldots\}$ depending on any neighborhood $V$ of $x$ such that $\left|\mu_{n_{k}}\right|(V) \geq \varepsilon$. If $K$ is a compact subset of the deleted neighborhood $V$ of $x$, then $V \sim K$ is a neighborhood of $x$, and so there is another subsequence $\left\{\mu_{p_{k}} \mid k=1,2, \ldots\right\}$ such that $\left|\mu_{p_{k}}\right|(V \sim K) \geq \varepsilon$. This is the necessary device. Q.E.D.

This proposition enables us to prove a special instance of the desired result.

Proposition 3.2. Let the hypotheses of Proposition 3.1 hold, and assume further that for each Borel set $E$, set function $\mu_{n}$ and $\varepsilon>0$, there exists a countable compact set $K \subseteq E$ such that $\left|\mu_{n}(E \sim K)\right|<\varepsilon$. Then $\lim _{n \rightarrow \infty}\left|\mu_{n}\right|(X)=0$. 
Proof. By Proposition 2.3 we need only show that, for every compact set $K$ used in the proof of Proposition 2.3, $\lim _{n \rightarrow \infty}\left|\mu_{n}\right|(K)=0$. It is not difficult to show that all such compact sets can be chosen to be countable, so let $K=\left\{x_{n} \mid n=1,2, \ldots\right\}$. For each integer $n$, let $\left\{N\left(x_{n}, \varepsilon / 2^{n}\right) \mid n=1,2, \ldots\right\}$ and $\left\{U\left(x_{n}, \varepsilon / 2^{n}\right) \mid n=1,2, \ldots\right\}$ be the integers and neighborhoods of Proposition 3.1. Then $K \subseteq \cup_{n=1}^{\infty} U\left(x_{n}, \varepsilon / 2^{n}\right)$; by compactness there is an integer $N$ such that $K \subseteq \cup_{n=1}^{N} U\left(x_{n}, \varepsilon / 2^{n}\right)$. So

$$
\begin{aligned}
k & \geq \max \left(N\left(x_{1}, \varepsilon / 2\right), \ldots, N\left(x_{N}, \varepsilon / 2^{N}\right)\right) \\
& \Rightarrow\left|\mu_{k}\right|(K) \leq\left|\mu_{k}\right|\left(\bigcup_{n=1}^{N} U\left(x_{n}, \varepsilon / 2^{n}\right)\right) \\
& \leq \sum_{n=1}^{N}\left|\mu_{k}\right|\left(U\left(x_{n}, \varepsilon / 2^{n}\right)\right)<\varepsilon,
\end{aligned}
$$

completing the proof. Q.E.D.

There are two simple examples of when the foregoing is applicable-a space $X$ in which each compact set is countable, and whenever all the set functions are atomic.

Of course, the optimal situation would be to be able to show that for each point $x$ there is a neighborhood $U(x)$ of $x$ such that $\lim _{n \rightarrow \infty}\left|\mu_{n}\right|(U(x))=0$. Then clearly $\lim _{n \rightarrow \infty}\left|\mu_{n}\right|(K)=0$ for each compact set $K$, and Proposition 3.3 applies. Essentially, Proposition 3.2 is merely an instance in which $\lim _{n \rightarrow \infty}\left|\mu_{n}\right|(K)=0$ can be easily deduced.

In regard to Proposition 2.4, a theorem of Grothendieck (Sur les applications linéaires faiblement compactes d'espaces du type $C(K)$, Canad. J. Math. 5(1953), 129-173) states that, if $\left\{\mu_{n} \mid n=1,2, \ldots\right\}$ is a sequence of regular Borel measures defined on the Borel subsets of a compact Hausdorff space such that $\mu_{n}(E) \rightarrow 0$ for each open set $E$, then $\mu_{n}(E) \rightarrow 0$ for all Borel sets. Coupling this with Alexandroff's theorem that regular set functions defined on compact sets are measures and the classical Vitali-Hahn-Saks theorem, we see that compact sets are admissible, and consequently this assumption is superfluous.

Additionally, in the Phillips' lemma case, the maximal open set may be empty. The author would like to thank Professor William G. Bade for the following example: let $X=[0,1]$ with the usual topology. Divide it into $2^{n}$ intervals of equal length, and let $f_{n}$ be 1 on the odd intervals and -1 on the even ones. Let $m$ denote Lebesgue measure, and define $\mu_{n}(E)=\int_{E} f_{n} d m$. Then $\mu_{n}(E) \rightarrow 0$ for each Borel set, but $\left|\mu_{n}\right|(E)=m(E)$ for each open set $E$.

\section{BIBLIOGRAPHY}

1. R. S. Phillips, On linear transformations, Trans. Amer. Math. Soc. 48 (1940), 516-541. MR 2, 318.

2. J. D. Stein, Jr., A uniform boundedness theorem for measures, Michigan Math. J. 19 (1972), 161-165.

Department of Mathematics, University of California, Los Angeles, California 90024

Department of Mathematics, Århus University, Århus, Denmark 